\title{
Feline Sino-orbital Fungal Infection Caused by Aspergillus and Scopulariopsis
}

\author{
Fernanda Vieira Amorim da Costa' ${ }^{1}$ Andreia Spanamberg ${ }^{2}$, Ricardo Araujo ${ }^{3,4}$, Juliana Werner ${ }^{5}$ Laerte Ferreiro ${ }^{2}$
}

\begin{abstract}
Background: Deep fungal infections of the orbit and nasal passages causing rhinitis and ulcerative keratomycosis are uncommonly reported in cats. Hyalohyphomycetes and phaeohyphomycetes have rarely been associated with this disorder. Sino-orbital fungal diseases are emerging and more invasive than sino-nasal fungal diseases with poor response to therapy and a worse prognosis. Brachycephalic feline breeds seem to be at increased risk for development of upper respiratory fungal diseases. Diagnosis is based on the demonstration of fungal hyphae by cytology or histology and definitive confirmation by fungal culture and molecular methods. This is the first case report of a cat with clinical mixed fungal ball with Aspergillus and Scopulariopsis in Brazil.

Case: A 3-year-old male Persian cat, in São José city, Santa Catarina, Brazil, was presented with exophthalmos and corneal ulcer of the left eye and protrusion, hyperemia, quemosis and fibroses of the left third eyelid. The retropulsion of the globe was negative in this eyeball and a presumptive diagnosis of a retrobulbar mass was made. The patient underwent a surgical procedure for inspection and collection of samples for bacterial and mycological culture. Culture revealed no bacterial growth, however, unique and abundant growth of Aspergillus spp. was present. A subconjunctival enucleation of the left eye was made and the mass was sent for histopathology examination. Histology showed inflammatory proliferative necrotizing pyogranulomatous reaction; with the presence of severe fungal infection evidenced by large number of hyaline septated regular and irregular mold hyphae. Molecular identification was performed using panfungal primers (ITS3-F / ITS4-R). Patient was treated with systemic itraconazole associated with amphotericin B and topical clotrimazole. A mass started to grow rapidly in the left pterygopalatine fossa and was surgically removed, but recurrence occurred seven days after. After 22 days of treatment, the animal died suddenly with a history of acute inspiratory dyspnea and cyanosis at the time prior to death. The diagnosis of sino-orbital fungal disease in the feline was based on clinical signs, mycological culture, histopathology and molecular methods.

Discussion: Sino-orbital fungal diseases rare in cats and can result in significant injuries to the upper respiratory tract and eyes, sometimes resulting in enucleation and death. It seems feasible that a brachycephalic facial conformation may be an important risk factor for the development of sino-nasal fungal diseases in cats. Despite using selected drugs and eye enucleation to treat the disease, the cat developed a rapid growing oral mass that probably caused acute inspiratory dyspnea and death. Since no controlled studies exist on the treatment of feline fungal diseases, these cases are a challenge to the feline practitioner and this type of clinical manifestation should be included in the differential diagnosis of upper respiratory and ocular diseases.
\end{abstract}

Keywords: Aspergilllus, cat, fungal disease, retrobulbar mass, upper respiratory disease.

${ }^{1}$ Setor de Medicina Felina do Hospital de Clínicas Veterinárias (HCV) \& ${ }^{2}$ Setor de Micologia Veterinária, Faculdade de Veterinária (FaVet), Universidade Federal do Rio Grande do Sul (UFRGS), Porto Alegre, RS, Brazil. ${ }^{3}$ i3S - Instituto de Investigação e Inovação em Saúde, Porto, Portugal. ${ }^{4}$ Department of Medical Biotechnology, College of Medicine and Public Health, Flinders University, Adelaide, South Australia, Australia. ${ }^{5}$ Werner \& Werner - Laboratório de Patologia Veterinária, Curitiba, PR, Brazil. CORRESPONDENCE: F.V.A. Costa [fernanda.amorim@ufrgs.br - Tel.: +55 (51) 3308-9643]. Setor de Medicina Felina - HCV, UFRGS. Av. Bento Gonçalves n. 9090. Bairro Agronomia. CEP 91540-000 Porto Alegre, RS, Brazil. 


\section{INTRODUCTION}

Sino-nasal and sino-orbital fungal disease can be caused by hyalohyphomycetes (hyaline septate hyphae) and phaeohyphomycetes (pigmented septate hyphae). Investigations suggested that the majority of infections were caused by Aspergillus species. In cats, the disease occurs in two main forms: sino-nasal aspergillosis (SNA) and sino-orbital aspergillosis (SOA) $[5,16,29]$. SNA is characterized by signs of chronic nasal infection, such as sneezing, uni or bilateral serous to mucopurulent nasal discharge, and sometimes epistaxis. SOA is the more invasive form because the fungus invades the submucosal tissue of the nasal cavity and sinus and extends through the orbital bone and into the retrobulbar space. SNO clinical signs include unilateral exophthalmos, third eyelid prolapse, conjunctival hyperaemia and keratitis. Response to therapy is less successful in SNO aspergillosis and the prognosis is generally worse [16].

Definitive diagnosis requires a combination of imaging, visualization of fungal plaques by rhinoscopy, and identification of fungal structures on culture and cytology, or histopathology [4,29]. Treatment of this form of the disease should be multifactorial, including orbitotomy or eye enucleation and a combination of systemic antifungal treatment with local therapy using intranasal infusions under general anesthesia [16].

This is the first case report of a cat with clinical mixed fungal infection with Aspergillus and Scopulariopsis in Brazil showing details of the clinical presentation, diagnosis, medical and surgical management of the disease.

\section{CASE}

A 3-year-old male Persian cat, weighing $3.8 \mathrm{~kg}$ was seen at Green Cross Veterinary Clinic, São José, Santa Catarina, Brazil, presenting with exophthalmos, lagophthalmos and asymmetry of the frontal and dorsal face. Severe protrusion, hyperemia, chemosis and fibrosis of the left third eyelid were noted (Figure 1). The cat had normal vision in both eyes; vision was diminished in the left eye showing limited response to the menace reflexe in this eyeball. The Schirmer I Tear Test (STT strips) ${ }^{1}$ was $16 \mathrm{~mm} / \mathrm{min}$ in the right eye and $3 \mathrm{~mm} / \mathrm{min}$ in the left eye. Intraocular pressure (IOP) was measured using applanation tonometry (Tonopen $\left.{ }^{\circledR} \mathrm{XL}\right)^{2}$, after application of a topical ophthalmic anesthetic solution $\left(\text { Anestalcon }^{\circledR}\right)^{3}$. The IOP was 17 $\mathrm{mmHg}$ in the right eye and $48 \mathrm{mmHg}$ in the left eye.
However, the retropulsion of the globe was negative in this eyeball. The left superior cornea had an irregular form, corneal edema, neovascularization and retained fluorescein stain (Fluoresceína Strips $\left.{ }^{\circledR}\right)^{4}$ [Figure 2]. A presumptive diagnosis of a retrobulbar mass was made. Furthermore, the cat had mucopurulent nasal and ocular discharge from the same side.

The patient had no fever or weight loss, but had a history of poor appetite and prostration. Immunoassays for the detection of feline immunodeficiency virus and feline leukemia virus (FIV/FeLV) were negative. Complete blood count and serum biochemical assay were also performed, which showed only leukopenia and lymphopenia. Initial supportive therapy consisted of fluid and electrolyte therapy, eye drops of flurbiprofen, moxifloxacin and chondroitin sulfate, in addition to oral amoxicillin and cyproheptadine for 15 days.

After clinical improvement, the patient underwent a surgical procedure for inspection and collection of samples for cytology, culture, antimicrobial sensitivity profile and histopathological analysis of the mass. During the procedure, there was a purulent and caseous tissue in the third eyelid conjunctiva, occupying the medial and retrobulbar region (Figure 3).

Cytology showed the presence of red blood cells, predominance of degenerate neutrophils and few lymphocytes, and free and intracellular bacteria. Tissue fragments were subjected to aerobic culture at $37^{\circ} \mathrm{C}$ on blood agar and MacConkey agar, and incubated at $30^{\circ} \mathrm{C}$ on Sabouraud dextrose agar. Culture revealed no bacterial growth, however, unique and abundant growth of Aspergillus spp. was present.

The patient was then submitted to radiographic investigation, which showed increased radiopacity in the left sinus and loss of definition of the turbinate bones. The cat underwent a surgical procedure for subconjunctival enucleation of the left eye (Figure 4), which was sent for histopathology examination. Histology showed inflammatory proliferative necrotizing pyogranulomatous reaction, with presence of septate branching hyphae both regular and irregular, morphologically compatible with Aspergillus sp. and other hyaline septated mold (Figure 5). Special staining with Ziehl Nielsen techinique was performed for mycobacteria investigation, but the result was negative.

The DNA was extracted from the formalin fixed paraffin embedded (FFPE) sample using the QIAamp ${ }^{\circledR}$ FFPE tissue kit (Qiagen) ${ }^{5}$. DNA extracted 
was detected with panfungal PCR using ITS3-F (5'-GCATCGATGAAGAACGCAGC-3') and ITS4R (5' - TCCTCCGCTTATTGATATGC-3' [34] for amplification of internal transcribed spacer 2 (ITS2) region. PCR amplification was performed in a $25 \mu \mathrm{L}$ containing $1 \mu \mathrm{L}$ of DNA extract, $12.5 \mu \mathrm{L}$ Qiagen Taq PCR master mix (Qiagen) ${ }^{5}$ and $0.5 \mu \mathrm{L}$ of each primer (for a $0.2 \mu \mathrm{M}$ final concentration of each primer). After a preincubation at $94^{\circ} \mathrm{C}$ for $15 \mathrm{~min}$, the amplification was performed for a total of 35 cycles as follows: denaturation at $94^{\circ} \mathrm{C}$ for $30 \mathrm{~s}$, annealing at $57^{\circ} \mathrm{C}$ for 90 $\mathrm{s}$, extension at $72^{\circ} \mathrm{C}$ for $1 \mathrm{~min}$, and a final extension step of $10 \mathrm{~min}$ at $72^{\circ} \mathrm{C}$. The primers ITS3 and ITS4 amplify a fragment of between 300 and $400 \mathrm{bp}$. PCR product was separated on a $2 \%$ agarose. PCR product was purified using PuriLink ${ }^{\circledR}$ PCR Purification Kit (Invitrogen) $^{6}$, and sequencing to confirmed the presence of fungal elements in the tissue sample.

The sequencing analysis of the fragments revealed a clear mix of two fungal sequences (two peaks for each position). Aspergillus was initially cultured from the tissue, therefore the consensus profile for ITS2 of 100 Aspergillus sequences was subtracted from the sequencing graph to obtain the sequence for the second fungus (e.g. for a sequencing position with $\mathrm{A}$ and $\mathrm{C}$, if the nucleotide of consensus Aspergillus sequence was A, then the second position was considered to be C). At the end of this detailed analysis of each nucleotide position, two sequences were obtained. Basic local alignment search tool $\left(\text { BLAST }^{\circledR}\right)^{7}$ was performed in both sequences giving the results $100 \%$ match for $A s$ pergillus and Scopulariopsis respectively. No fungal species could be identified using these ITS 2 sequences, as these region was common (>99\% match) to multiple species within each genus.

The patient was treated with intranasal clotrimazole once a week and systemic itraconazole at a dose of $50 \mathrm{mg}$ orally per day. After 20 days of treatment, the patient showed a mass of $2 \mathrm{~cm}$ in diameter on the left side of the soft palate (Figure 6A), which was removed surgically. However, recurrence occurred 7 days after surgery (Figure 6B), and the patient began to show labored breathing due to obstruction of the oropharynx.

Due to the rapid evolution of the disease and poor response to treatment, the cat was treated with subcutaneous amphotericin B at $0.5 \mathrm{mg} / \mathrm{kg}$ diluted in $350 \mathrm{~mL}$ of warm $0.45 \%$ sodium chloride and $2.5 \%$ glucose, and intravenous fluid therapy with lactated
Ringer's solution at the maintenance dose of $50 \mathrm{~mL} /$ $\mathrm{kg} /$ day. After 2 days of treatment, the animal died suddenly with a history of acute inspiratory dyspnea and cyanosis at the time prior to death. The animal's owner declined to perform a necropsy.

\section{DISCUSSION}

Sino-orbital fungal disease is rare in cats and can result in significant injuries to the upper respiratory tract and eyes, sometimes resulting in enucleation and death. In this report, the patient was a Persian cat, agreeing with previous reports that 8 out of 12 cats treated for nasal or ocular aspergillosis were Persian or Himalayan $[2,12,15,18,23,31,35]$. It seems feasible that a brachycephalic facial conformation may be an important risk factor for the development of sino-nasal fungal disease in cats [29], because fungal organisms are a part of the normal feline ocular and nasal flora $[6,19]$ and it is possible that the brachycephalic airway of Persian and Himalayan cats may at times become overwhelmed in its ability to clear Aspergillus spp. from the cat's upper airway [2]. Tomsa et al. [31] presupposed that susceptibility to localized fungal infection can occur as a consequence of the possible altered airflow observed in pronounced brachycephaly. This could cause changes in the vasomotor action of the vasculature and decrease mucociliary clearance. Wilkinson et al. [36] reported two cats with sino-nasal disease who also had involvement of the orbit. In one case this was associated with fungal sinusitis. Our patient had both presentations of this disease, with the same clinical signs as reported for the sino-orbital (SOA) and SNA forms. The disease probably extended beyond the sinonasal cavity and involved the adjacent orbital structure and palate. Therefore, the first presenting signs in cats are referable to orbital invasion [3]. In this case, the patient presented first orbital involvement, and the signs were exophthalmos, high intraocular pressure, severe protrusion and inflammation of the third eyelid. All of these signs were likely secondary to an invasive retrobulbar fungal granuloma that was pushing and compressing the eyeball, characterizing the SOA form $[2,3,15,35]$. Furthermore, the patient showed mucopurulent nasal and ocular discharge from the same side, corroborating with Hamilton et al. [15] and Barachetti et al. [2], which said that these signs are commonly found in nasal aspergillosis. The corneal and lacrimal film alterations, including the corneal 


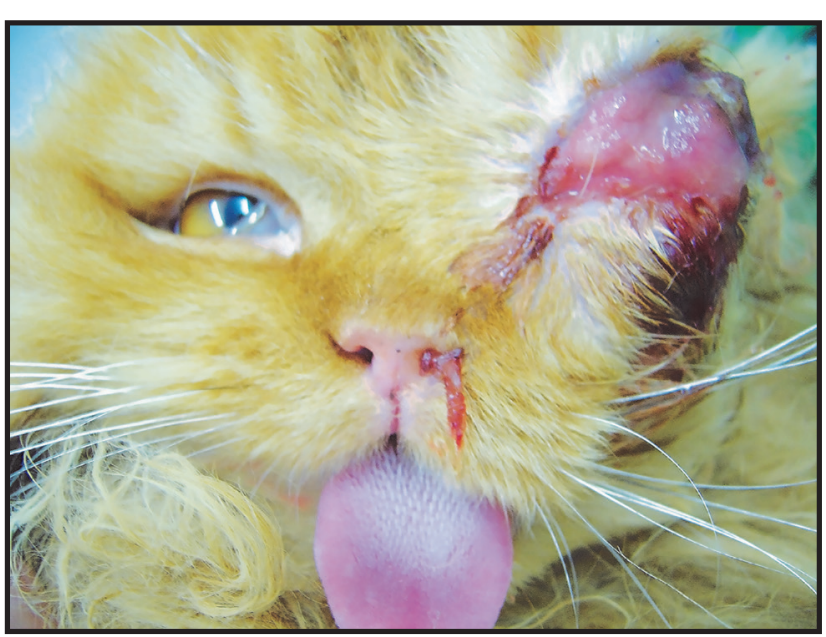

Figure 1. Patient showing protrusion, hyperemia, chemosis of the third eyelid and exophthalmos in the left eye. It's possible to note nasal and ocular mucopurulent discharge in the same side. ulcer and low STT in the left eye, were possibly due to corneal exposure, a consequence of the exophthalmos and lagophthalmos [2,5,15,35].

Disseminated aspergillosis is apparently an opportunistic infection in immunosuppressed cats [26]. In contrast, incompetence of the immune system is not believed to be required for nasal infection. Only 2 of 16 previously reported cats with sinonasal or orbital aspergillosis had a confirmed immunosuppressive condition $[12,15,18,23,31,35]$. In this study, the animal had no history of trauma or previous infections and was negative for FIV and FeLV infection, both important causes of immunosuppression in cats.

Diagnostic imaging for orbital disease typically includes skull radiography and orbital ultrasonography, however, CT and magnetic resonance imaging (MRI) have been successfully used to aid in the diagnosis of

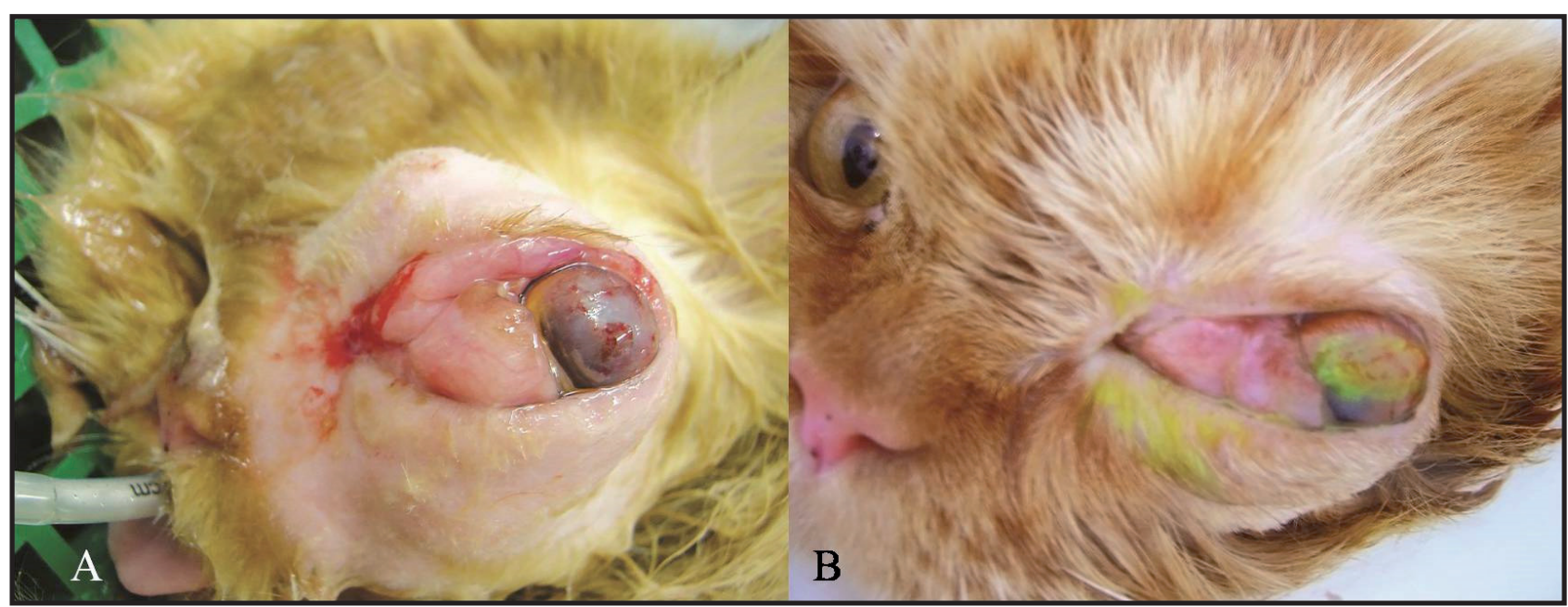

Figure 2. A- Lagophthalmos, corneal edema, neovascularization and mydriasis in the left eye. B- Visible corneal ulcer after the lesion retained fluorescein stain.

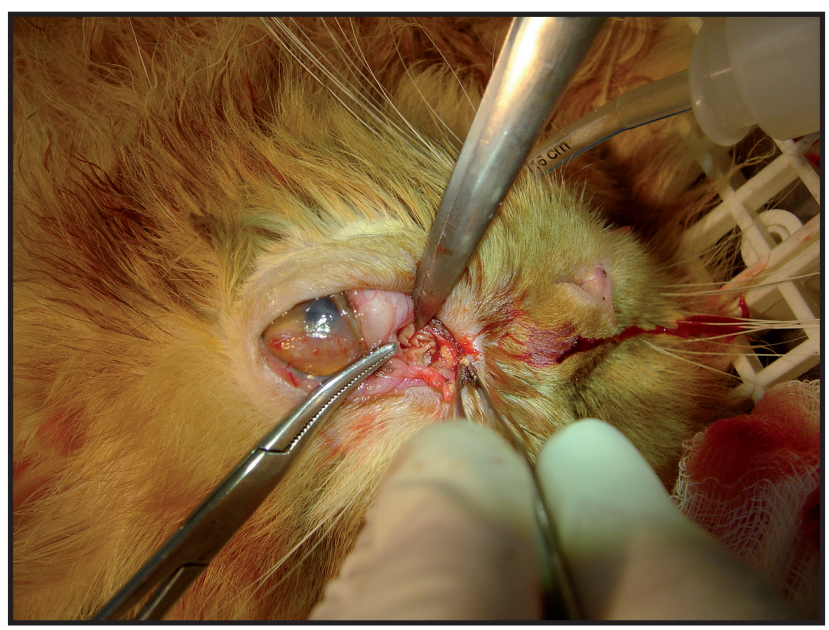

Figure 3. Inspection and biopsy of medial region of the left eye showing purulent and caseous tissue.
Aspergillus spp. infections [15,16,25,29]. Radiography of the nasal cavity and frontal sinuses may show abnormalities such as increased soft-tissue densities, loss of turbinates $[9,16,31,35]$ and possible bone erosion/lysis [2,9], as the patient showed in this report. CT imaging is important for assessing the extent of the disease [35] because of its ability to generate cross-sectional images [8]. This technique is also useful to detect calcium accumulation within the fungus colony or bone destruction [25]. However, MRI is the best technique to diagnose soft tissue lesions and vascular invasion $[16,25]$. It can be used to evaluate more critically the optic chiasm, differentiate neoplasia and determine the full extent of the disease [2,25]. Occasionally, cytology of nasal discharge or nasal flush will show septate hyphae. The presence of hyphae in the tissues 
F.V.A. Costa, A. Spanamberg, R. Araujo, J. Werner \& L. Ferreiro. 2019. Feline Sino-orbital Fungal Infection Caused by Aspergillus and Scopulariopsis.

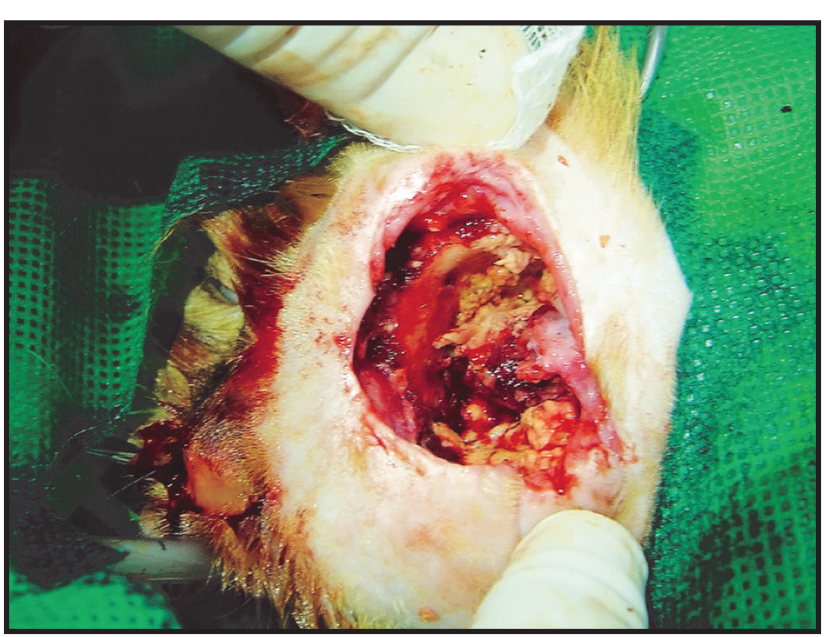

Figure 4. Left orbital cavity after the enucleation with purulent and caseous tissue.

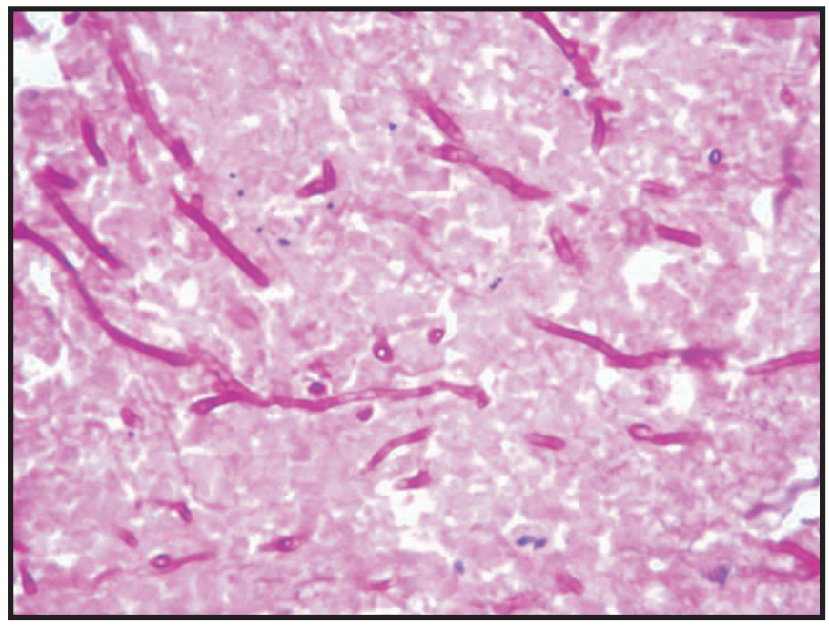

Figure 5. Microscopy of sino-orbital lesion showing necrotic tissue with multiple irregular, septate branching hyphae, morphologically compatible with Aspergillus sp. and other hyaline septated mold [PAS; 200x].

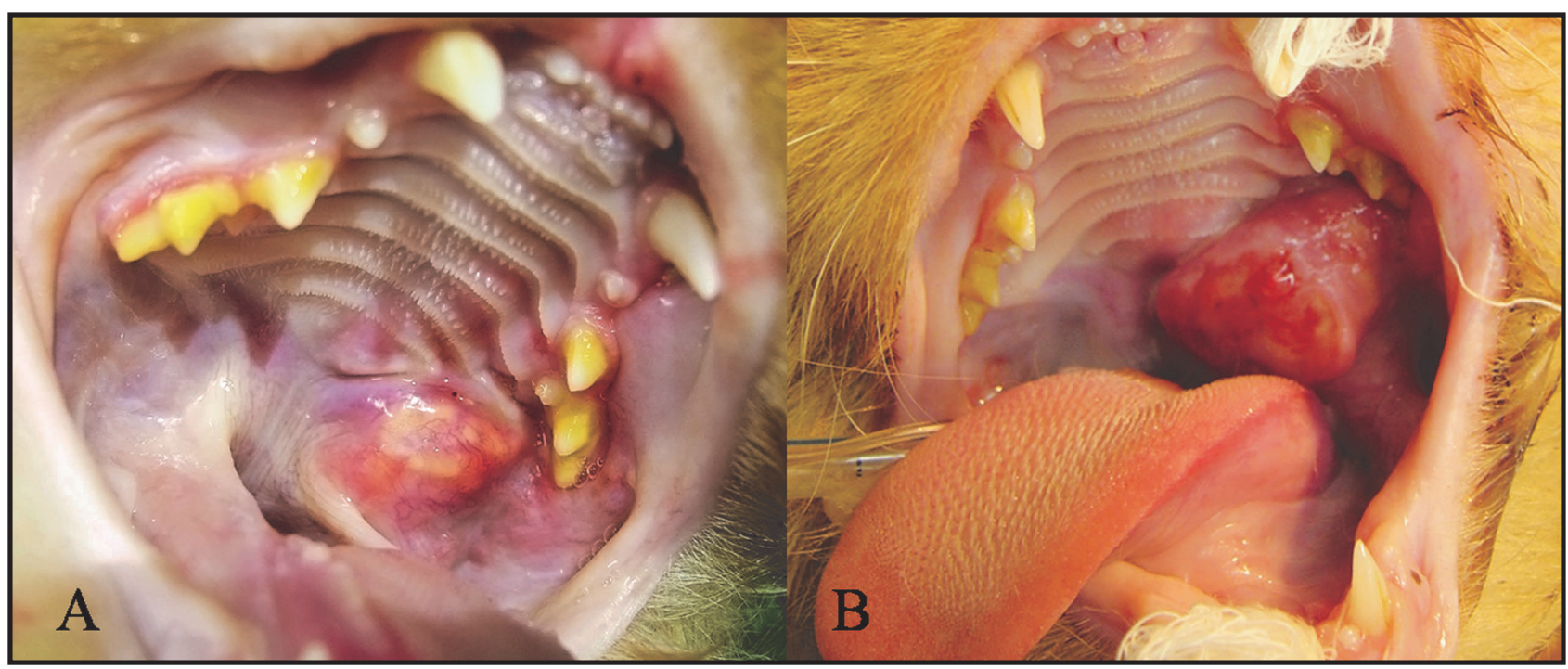

Figure 6. After treatment with antifungal therapy. A- Mass of $2 \mathrm{~cm}$ in diameter in the soft palate on the left side. B- Recurrence of the lesion in the soft palate 7 days after surgery.

will aid in differentiating diagnosis of Aspergillus spp. from Mucor spp., and Candida spp. Infections [9]. However, sometimes it can be inconclusive [15,23], as in the case reported here.

Occasionally, the results of fungal culture do not distinguish between Aspergillus spp. colonization and active infection [26]. False negatives can also occur [36]. Nevertheless, when a sample can be obtained directly from the site of visible fungal infection, a positive fungal culture would provide useful adjunct diagnostic information $[23,31]$. In this report, culture from biopsied material revealed unique and abundant growth of Aspergillus spp.

In this case, histology showed inflammatory proliferative necrotizing pyogranulomatous reaction, with the presence of severe fungal infection evidenced by septate hyaline hyphae (suggesting both regular and irregular structures), some with dichotomous branching suggestive of Aspergillus sp. corroborating with previous reports $[2,17,31,35]$. Histopathology has been used as the primary ante mortem diagnostic method of feline aspergillosis [10]. Biopsy samples are more likely to reveal the organisms, but false negative biopsies occur if only the superficial aspect of the lesion is biopsied [35].

Molecular methods are needed for correct fungal identification when the conventional methodologies (histopathology and culture) does not allow the complete clarification. In our study, although the Aspergillus sp. has grown in culture, PCR panfungal was required to confirm fungal identification and a possible case of co-presence of molds, due to histopathology suggesting 
multiple fungi. The presence of another fungus, Scopulariopsis, was confirmed by sequencing analysis of ITS2. In tissue the features of hyaline septated molds were similar to those seen with aspergillosis and Scopulariopsis mycosis [13].

The most common causes of chronic nasal discharge in cats include nasal neoplasia, fungal sinorhinitis -Aspergillosis [6] and cryptococcosis [21,32] - upper respiratory complex [1,5,30,33] and bacterial, allergic and idiopatic rhinitis [9]. The drugs available to treat fungal infections of the eyeball are restricted because eyedrops have limited penetration into the eyeball [11]. One option is topical itraconazole $1 \%$ which may be useful to treat corneal ulcers caused by Aspergillus fumigatus [22]. In this case, only supportive therapy was prescribed until a definitive diagnosis was made, that's why a systemic treatment was preferably chosen.

Itraconazole generally has good in vitro activity against Aspergillus spp., and itraconazole-resistant strains are uncommonly seen [24].Azoles are by far the most ineffective agents, and amphotericin B has limited activity against Scopulariopsis spp. [27,28]. In this patient, oral itraconazole was initially selected because of its documented efficacy against a variety of deep mycotic infections in cats and its relative safety in this species [7]. But its effectiveness for treatment of aspergillosis has controversial results. Itraconazole was effective in four cats with SNA aspergillosis, but three of them had recurrence of clinical signs following discontinuation of treatment $[31,35]$. However, itraconazole was ineffective for a cat with orbital aspergillosis [24]. In two studies of SOA, there was no clinical improvement with use of itraconazole and then amphotericin B systemically [18,23]. Amphotericin B has been used for more than 40 years in aspergillosis treatment and also targets the fungal cell membrane. Its less toxic lipid formulations have been used in cases of aspergillosis refractory to azole antifungals [20]. Resistance to AMB is a rare phenomenon in $A$. fumigatus, however A.terreus is intrinsically resistant and $A$. flavus has reduced sensitivity to this antifungal [14]. Topical therapy with a non-invasive infusion of clotrimazole is considered the treatment of choice for canine nasal aspergillosis. The cure rate has been as high as 94\%.28 This technique has been reported effective in three cats with fungal rhinitis [12,31]. Two other cats were treated with a topical infusion of clotrimazole in previous reports $[15,23]$. One had no response to treatment and the other responded initially but was euthanatized later because of the development of new clinical signs of dysphagia and anorexia. Both of these cats had also SOA, similar to the case reported here, while the cats that responded successfully to clotrimazole had SNA.

In this case, the animal died suddenly. Certainly, the brachycephalic conformation decreases sinus aeration and drainage of respiratory secretions secondary to infection, polyps, and allergic rhinosinusitis, which have been identified as risk factors for invasive SNA aspergillosis in human beings [3]. As was observed, the patient had a mass in the soft palate, which was causing the difficulty to breath and feeding. This was probably the reason for death, because he showed acute inspiratory dyspnea and cyanosis at his final hours.

Orbital aspergillosis/hialohyphomycosis is rare in cats and can result in significant injuries to the eyes, sometimes resulting in enucleation. Nasal aspergillosis is also uncommon and can cause dyspnea and death of cats through nasopharyngeal asphyxia. The association of both forms can occur and, unfortunately, the treatment is ineffective so far. Further studies are required in order to better understand and efficiently treat fungal sino-orbital infections in this species.

\section{MANUFACTURERS}

${ }^{1}$ Schering Plough Anima Health. Union, NJ, USA.

${ }^{2}$ Mentor O \& O Inc. Norwell, MA, USA.

${ }^{3}$ Allergan Pharmaceuticals. São Paulo, SP, Brazil.

${ }^{4}$ Ophthalmos Pharmaceuticals. São Paulo, SP, Brazil.

${ }^{5}$ Qiagen. Hilden, Germany.

${ }^{6}$ Invitrogem. Carlsbad, CA, USA.

${ }^{7}$ U.S. National Library of Medicine. Rockville, MD, USA.

Declaration of interest. The authors report no conflicts of interest. The authors alone are responsible for the content and writing of paper. 


\section{REFERENCES}

1 Andrew S.E. 2001. Ocular manifestations of feline herpesvirus. Journal Feline Medicine Surgery. 3: 9-16.

2 Barachetti L., Mortellaro C.M., Di Giancamillo M., Giudice C., Martino P., Travetti O. \& Miller P.E. 2009. Bilateral orbital and nasal aspergillosis in a cat. Veterinary Ophthalmology. 12: 176-182.

3 Barrs V.R., Beatty J.A., Lingard A.E., Malik R., Krockenberger M.B., Martin P., O’Brien C., Angles J.M., Dowden M. \& Halliday C. 2007. Feline sino-orbital aspergillosis: an emerging clinical syndrome. Australian Veterinary Journal. 85(3):23.

4 Barrs V.R. \& Beatty J.A. 2010. Upper respiratory tract aspergillosis. In: August J.R. (Ed). Consultations in Feline Medicine. 6th edn. St. Louis: Saunders Elsevier, pp.36-52.

5 Barrs V.R. \& Talbot J.J. 2014. Feline aspergillosis. The Veterinary Clinics of North America Small Animal Practice. 44(1): 51-73

6 Benitah N. 2006. Canine Nasal Aspergillosis. Clinical Techniques Small Animal Practice. 21: 82-88.

7 Boothe D.M., Herring I., Calvin J., Way N. \& Dvorak J. 1997. Itraconazole disposition after single oral and intravenous and multiple oral dosing in healthy cats. America Journal Veterinary Research.58: 872-877.

8 Choi M.Y., Bae I.H., Lee J.H. \& Lee S.J. 2002. Aspergillosis presenting as an optic neuritis. Korean Journal of Ophthalmology.16: 119-123.

9 Codner E.C., Lurus A.G., Miller J.B., Gavin P.R., Gallina A. \& Barbee D.D. 1993. Comparison of computed tomography with radiography as a noninvasive diagnostic technique for chronic nasal disease in dogs. Journal of the American Veterinary Medical Association. 202: 1106-1110.

10 Davies C. \& Troy G. 1996. Deep mycotic infections in cats. Journal of the American Veterinary Medical Association. 32: 380-391.

11 Freda R. 2006. Use of oral voriconazole as adjunctive treatment of severe cornea fungal infection: case report. Arquivos Brasileiros de Oftalmologia. 69: 431-434.

12 Furrow E. \& Groman R.P. 2009. Intranasal infusion of clotrimazole for the treatment of nasal aspergillosis in two cats. Journal of the American Veterinary Medical Association. 235: 1188-1193.

13 Guarner J. \& Brandt M.E. 2011. Histopathologic diagnosis of fungal infections in the 21st century - Clinical microbiology reviews. Journal of the American Veterinary Medical Association. 213: 501-506.

14 Gonçalves S.S., Souza A.C.R., Chowdhary A., Meis J.F. \& Colombo A.L. 2016. Epidemiology and molecular mechanisms of antifungal resistance in Candida and Aspergillus. Mycoses. 59: 198-219.

15 Hamilton H.L., Whitley R.D. \& McLaughlin S.A. 2000. Exophthalmos secondary to aspergillosis in a cat. Journal of the American Animal Hospital Association. 36: 343-347.

16 Hartmann K., Lloret A., Pennisi M.G., Ferrer L., Addie D., Belák S., Boucraut-Baralon C., Egberink H., Frymus T., Gruffydd-Jones T., Hosie M.J., Lutz H., Marsilio F., Möstl K., Radford A.D., Thiry E., Truyen U. \& Horzinek M.C. 2013. Aspergillosis in cats: ABCD guidelines on prevention and management. Journal of Feline Medicine and Surgery. 15(7): 605-610.

17 Hazell K.L.A., Swift I.M. \& Sullivan N. 2011. Successful treatment of pulmonary aspergillosis in a cat. Australian Veterinary Journal. 89(3): 101-104.

18 Kano R., Itamoto K., Okuda M., Inokuma H., Hasegawa A. \& Balajee S.A. 2008. Isolation of Aspergillus udagawae from a fatal case of feline orbital aspergillosis. Mycoses. 51: 360-361.

19 Labelle A.L., Hamor R.E., Barger Anne M., Maddox C.W. \& Breaux C.B. 2009. Aspergillus flavus keratomycosis in a cat treated with topical $1 \%$ voriconazole solution. Veterinary Ophthalmology. 12: 48-52.

20 Linden P.K. 2003. Amphotericin B lipid complex for treatment of invasive fungal infections. Expert Opinion on Pharmacotherapy. 4: 2099-2110.

21 Malik R., Wigney D.I., Muir D.B. \& Love D.N. 1997. Asymptomatic carriage of Cryptococcus neoformans in the nasal cavity of dogs and cats. Journal of Medical and Veterinary Mycology. 35: 27-31.

22 Martínez-Ramos M., Claros-B J.A., Vale-Oviedo M.A., Siso-Villarroel E., Padilla R., Santiago A. \& Simón J.A. 2008. Effect of the vehicle on the topical itraconazole efficacy for treating corneal ulcers caused by Aspergillus fumigatus. Clinical and Experimental Ophthalmology. 36: 335-338.

23 McLellan G.J., Aquino S.M., Mason D.R., Kinyon J.M. \& Myers R.K. 2006. Use of posaconazole in the management of invasive orbital aspergillosis in a cat. Journal of the American Animal Hospital Association. 42: 302-307. 
24 Moore C.B., Sayers N., Mosquera J., Slaven J. \& Denning D.W. 2000. Antifungal drug resistance in Aspergillus. Journal of Infection. 41: 203-220.

25 Morgan R.V. 2011. Aspergillosis, Nasal (Zoonotic). Available at: www.vin.com/Members/Associate/Associate. plx?DiseaseId=2088. [Accessed online in February 2011].

26 Sharp N.J.H. 1998. Canine nasal aspergillosis-penicilliosis. In: Greene C.E. (Ed). Infectious diseases of the dog and cat. 2nd edn. Philadelphia: WB Saunders Co, pp.404-409.

27 Skóra M., Bulanda M. \& Jagielski T. 2015. In vitro activities of a wide panel of antifungal drugs against various Scopulariopsis and Microascus species. Antimicrobial Agents and Chemotherapy. 59(9): 5827-5829.

28 Skóra M., Macura A.B. \& Bulanda M. 2014. In vitro antifungal susceptibility of Scopulariopsis brevicaulis isolates. Medical Mycology. 52(7): 723-727.

29 Sykes J.E. 2014. Canine and Feline Infectious Diseases. St. Louis: Elsevier Saunders, 915p.

30 Thiry E., Addie D., Belák S., Boucraut-Baralon C., Egberink H., Frymus T., Gruffydd-Jones T., Hartmann K., Hosie M.J., Lloret A., Lutz H., Marsilio F., Pennisi M.G., Radford A.D., Truyen U. \& Horzinek M.C. 2009. Feline herpesvirus infection. ABCD guidelines on prevention and management. Journal Feline Medicine Surgery. 11: 547-555.

31 Tomsa K., Glaus T.M., Zimmer C. \& Greene C.E. 2003. Fungal rhinitis and sinusitis in three cats. Journal of the American Veterinary Medical Association. 222: 1380-1384.

32 Trivedi S.R., Sykes J.E., Cannon M.S., Wisner E.R., Meyer W., Sturges B.K., Dickinson P.J. \& Johnson L.R. 2011. Clinical features and epidemiology of cryptococcosis in cats and dogs in California: 93 cases (1988-2010). Journal of the American Veterinary Medical Association. 239: 357-369.

33 Volopich S., Benetka V., Schwendenwein I., Möstl K., Sommerfeld-Stur I. \& Nell B. 2005. Cytologic findings, and feline herpesvirus DNA and Chlamydophila felis antigen detection rates in normal cats and cats with conjunctival and corneal lesions. Veterinary Ophthalmology. 8: 25-32.

34 White T.J., Bruns T., Lee S. \& Taylor J. 1990. Amplication and direct sequencing of fungal ribosomal RNA genes for phylogenetics. In: Innis M.A., Gelfand D.H., Sninsky J.J. \& White T.J. (Eds). PCR protocols: a guide to methods and applications. San Diego: Academic Press Inc., pp.315-322.

35 Whitney B.L., Broussard J. \& Stefanacci J.D. 2005. Four cats with fungal rhinitis. Journal Feline Medicine Surgery. 7: $53-58$.

36 Wilkinson G.T., Sutton R.H. \& Grono L.R. 1982. Aspergillus spp. infection associated with orbital cellulitis and sinusitis in a cat. Journal of Small Animal Practice. 23: 127-131. 